\title{
Magnetophonon resonance in quantum wells in tilted field. What is concealed behind its angular dependence?
}

\author{
V. V. Afonin ${ }^{a, b}$, V. L. Gurevich ${ }^{a, b}$, R. Laiho ${ }^{a}$, M. O. Safonchik ${ }^{a, b}$, M. A. Shakhov ${ }^{a, b}$, \\ M. L. Shubnikov ${ }^{a, b}$ \\ ${ }^{a}$ Wihuri Physical Laboratory, University of Turku, FIN-20014 Turku, Finland \\ ${ }^{b}$ A. F. Ioffe Institute, 194021 Saint Petersburg, Russia
}

\begin{abstract}
Magnetophonon resonance in quantum wells in a tilted magnetic field $\mathbf{B}$ is investigated. Measurements of the Hall coefficient and correspondingly of the carrier concentration as a functions of magnetic field and temperature are simultaneously performed. It is shown that the experimental data can be interpreted in terms of a great sensitivity of the effect to the variation of the two dimensional carrier concentration $n_{s}$ in a certain concentration interval. In other words, the observed angular dependence of the MPR amplitudes is a manifestation of dependence of $n_{s}$ on the magnitude of the magnetic field $B$. PACS 73.50.Jt, 73.50.Mx, 63.20.Pw, 71.70.Di
\end{abstract}

\section{INTRODUCTION}

Magnetophonon resonance (MPR) in semiconductors is reached every time when the limiting frequency of a longitudinal optic phonon $\omega_{0}$ equals the cyclotron frequency of an electron, $\Omega$, times some small integer, $\mathcal{N}$ (see Refs. [1, 2]1). Along with cyclotron resonance, it has become one of the main instruments of semiconducting compound spectroscopy.

The advances in semiconductor nano-fabrication in recent years have made available nanostructures of a great crystalline perfection and purity. The electrical conduction and 
some other transport phenomena in such specimens has been a focus of numerous investigations, both theoretical and experimental. In particular, the discovery of MPR in the quantum wells took place in the pioneering paper by Tsui, Englert, Cho and Gossard [3]. The most detailed experimental investigation of MPR in quantum wells has been done by Nicholas and co-workers ( [4] and the references therein). It has been shown that there is a qualitative difference between MPR in 2D and 3D structures.

In the $2 \mathrm{D}$ case MPR can exist only in a relatively narrow interval of electron concentrations $n_{s}$. This has been indicated in Ref. [4] and explained qualitatively in Ref. [5]. In a special group of experiments [3,4] an angular dependence of MPR has been investigated. As is well known, the 2D magnetoconductance, including the MPR [6], at high magnetic fields $B$ should depend on the combination $B \cos \theta$ (see, for example, [7]). Here $\theta$ is the angle between the magnetic field $\mathbf{B}$ and the perpendicular to the plane of the well. One can easily understand this using the following classical analogy. In the 2D case the curvature of an electron's trajectory (in the course of electron's periodic motion in the plane) can be considered as nonexistent in the direction perpendicular to the plane because of the electron's interaction with the walls of the well. This means that all the physical quantities can depend only on the perpendicular component of the field. In particular, the position of the $\mathcal{N}$ th MPR is given by

$$
B_{\mathcal{N}}(\theta) \cos \theta=B_{\mathcal{N}}(0)
$$

where $B_{\mathcal{N}}(\theta)$ is the position of the MPR maximum for $\mathbf{B}$ directed at the angle $\theta$ to the perpendicular to the plane of the well while

$$
B_{\mathcal{N}}(0)=m \omega_{0} c / e \mathcal{N}
$$

Here $\omega_{0}$ is the limiting frequency of the optic phonons (we will not discriminate between the frequencies $\omega_{l}$ and $\omega_{t}$ - because of the insufficient accuracy of our experiment) and $m$ is the effective mass. Experimentally the angular dependence has been investigated by Tsui et al. [3] and Brummel et al. [8]. They have observed the angular dependence of the 
amplitude of MPR maximum that appeared to be very sharp whereas according to Eq. (四) the amplitude of MPR maximum should be independent of $\theta$ at all. That makes a drastic disagreement between the experiment and theory. This means that there is some feature in the system considered depending on the total magnetic field $B$ rather than on the combination $B \cos \theta$.

One of the main characteristics of the sample is the carrier concentration $n_{s}$. It is usually implied that it depends neither on the temperature nor on the magnetic field. Usually it is really so at low temperatures where most experiments with nanostructures are performed. However, the MPR experiments are made at relatively high temperatures, the highest amplitudes in GaAs being observed at $T$ about $180 \mathrm{~K}$ (they depend on $\mathcal{N}$ but only slightly). It is natural therefore to check the temperature and magnetic field dependence of the concentration. In order to control the electron concentration $n_{s}$, we have performed observation of MPR along with measurements of the Hall effect in 2D structures. Thus the purpose of the present paper is the investigation of the MPR, simultaneous measurement of magnetic field and temperature dependence of $n_{s}$ and interpretation of the obtained data.

\section{EXPERIMENTAL RESULTS}

Three series of $\mathrm{GaAs} / \mathrm{Al}_{x} \mathrm{Ga}_{1-x}$ As quantum well samples grown by molecular beam epitaxy were cut into a shape of a typical Hall bar for observation of the Shubnikov-de Haas (SdH) and the MPR oscillations. To avoid overheating of the sample during the magnetic

field pulse, we chose the measuring current to be sufficiently small (of the order of $5 \mu \mathrm{A}$ ). The measurements were carried out over the temperature interval of $4.2-300 \mathrm{~K}$ in pulsed magnetic fields up to $B=40 \mathrm{~T}$ with the pulse duration of $8 \mathrm{~ms}$. The main tool for collecting the data in our pulsed field installation is the data acquisition card with four fast independent $1 \mu \mathrm{s}, 12$ bit digital channels having $128 \mathrm{~Kb}$ buffer memory each.

The measured signal had a smooth nonlinear component with the amplitude much bigger than the amplitude of the investigated MPR oscillation. To single out the oscillation and to 
get rid of the high frequency noise we used the software package, based on the approximation of the curve by the polynomial minimum squares method with the Gaussian weight function. The method permits one to process the signals properly, particularly at the edges of the interval of magnetic field variation. However, it brings about some distortion of the form of oscillation, especially for the peaks near the edges (namely, the oscillation shifts towards smaller fields while its amplitude goes down). Nevertheless, if an edge of the interval is within the same phase of the MPR, the distortion of the last peak should be also the same for all the curves and the results can be compared. As under rotation of the specimen the maxima shift towards bigger fields [see Eq. (11)] the maximal pulse field $B_{\max }$ should also have the angular dependence $B_{\max } / \cos \theta$. All the rest parameters used for the processing remained the same for all the pulses.

Well developed SdH oscillations periodic in $1 / B$ were observed at $T=4.2 \mathrm{~K}$ and used to determine the values of the low-temperature carrier concentrations of the samples, namely $n_{s}=2.2,2.3$ and $4.0 \times 10^{11} \mathrm{~cm}^{-2}$. As pronounced MPR oscillations require sufficient optical phonon population they are usually observable at elevated temperatures. For this reason, we applied the Hall geometry to investigate the dependence of the carrier concentration on the applied field at fixed temperatures between 80 - $300 \mathrm{~K}$. Correspondingly, the temperature dependence of $n_{s}$ was determined between $80-300 \mathrm{~K}$ for $B$ between $0.95 \mathrm{~T}$ and $27 \mathrm{~T}$ (see Figs. 1 and 2).

The MPR oscillations, also periodic in $1 / B$, were recorded at different temperatures in the range $T=170-230 \mathrm{~K}$. In these measurements the magnetic field was tilted at an angle $\theta$. From the data the amplitude and the field positions of the $\mathcal{N}=2, \mathcal{N}=3$ and $\mathcal{N}=4$ MPR oscillation peaks were analyzed (for $\mathcal{N}=2$ and $\mathcal{N}=3$ they are given in Figs. $3-5$ ). Depending on the sample and the temperature, the accuracy of the results varied between $2-5$ per cent.

The magnetic field interval $6-15 \mathrm{~T}$ is important for our purpose. At the same time, the Hall resistance $\rho_{x y}$ shows a good linear dependence on the magnetic field already at $1 \mathrm{~T}$ (see Fig. 1), and the resonance at $\mathcal{N}=4$ corresponding to $5.62 \mathrm{~T}$ is reliably observed. This shows 
that for $B \gtrsim 6 \mathrm{~T}$ concentration variation as a function of magnetic field $B$ is rather big and the deviation from the linear dependence of $\rho_{x y}(B)$ cannot be explained by the corrections proportional to $(\Omega \tau)^{-2}, 1 / \tau$ being the collision frequency of the conduction electrons.

In the relevant magnetic field interval $6-12 \mathrm{~T}$ the rate of the electron concentration variation is temperature dependent (see Fig. 1). It is about 1.5 per cent per $1 \mathrm{~T}$ at $170 \mathrm{~K}$ and 3 per cent per $1 \mathrm{~T}$ at $200 \mathrm{~K}$. From the low temperatures up to $140 \mathrm{~K}$ there is no noticeable magnetic field dependence of concentration. This behavior fully correlates with the temperature dependence of the Hall coefficient. One can see a rather strong temperature dependence up from $140 \mathrm{~K}$. Mark that when either the temperature or magnetic field goes up the concentration $n_{s}$ also goes up.

\section{DISCUSSION OF THE RESULTS}

As one can expect, the relative rate of concentration variation with the temperature (see Fig. 2) is bigger than with magnetic field. The latter, however, is by a factor of $5-6$ bigger as compared with the concentration variation due to the spin magnetic moment of the free electrons. (The well-known corrections to the $g$-factor of electrons in quantum wells [9] can be disregarded as they also depend only on $B \cos \theta[10]$ ). This makes one think of the electrons that tunnel from discrete levels into a quantum well. Usually the electron levels go down with the magnetic field $B$. Indeed, as $B$ goes up the electron wave functions become nearer to the nuclei and therefore their binding becomes more tight or, in other words, the absolute value of the electron binding energy goes up. As a result, the level goes down as well as the electron concentration $n_{s}$ in the well. One of the possible mechanisms where the absolute value of the electron binding energy goes up with the magnetic field can be described as follows. If one has a negative ion where the electron is bound to the atom by dipole forces the magnetic field compression of the electron wave function should decrease the interaction with the atom. As a result, the probability that the electron will not be bound at elevated temperatures enhances. This means that the electron levels go up with 
the magnetic field and $n_{s}$ behaves in the same manner. Such ions could be either in the cover layer of the structure or in the interface.

In the papers by Nicholas et al. [4] a very strong dependence of the MPR amplitude was demonstrated in the region of high electron concentrations. The MPR amplitude started to go down at $n_{s}=10^{11} \mathrm{~cm}^{-2}$. At $n_{s}=3 \cdot 10^{11} \mathrm{~cm}^{-2}$ the decrease became extremely strong. The amplitude decreased by a factor 12 under the increase of concentration from $n_{s}=3 \cdot 10^{11} \mathrm{~cm}^{-2}$ to $n_{s}=5.5 \cdot 10^{11} \mathrm{~cm}^{-2}$.

This behavior is surprising. Indeed, the usual estimate of the relative role of electronelectron (e-e) interaction as compared with the kinetic energy of electrons gives

$$
e^{2} n^{1 / 2} / \varepsilon k_{\mathrm{B}} T
$$

This quantity is of the order of $1 / 4$ at $n_{s}=10^{11} \mathrm{~cm}^{-2}$ and $T=200 \mathrm{~K}$, i.e. it seems that one can neglect the e-e interaction. The degeneracy parameter becomes of the order of 1 at $n_{s} \approx 3 \cdot 10^{11} \mathrm{~cm}^{-2}$ [4]. However usually the onset of degeneracy changes the effect by something like a factor 2 , or so, whereas a much more substantial variation with the electron concentration was actually observed.

The physics of such a behavior has been described in Ref. [5]. Qualitatively it can be interpreted in the following way. Usually one interprets MPR as a result of electron transitions between two Landau levels. However another, a less direct approach is also possible. One can treat MPR as enhancement of interaction of a pair of electrons due to exchange of an optic phonon (a pole of the scattering amplitude). One should, however, take into consideration that, apart from the interaction due to exchange of a phonon, the electrons have also a direct Coulomb interaction. The sum of these two interactions can be described by a potential [11]

$$
V=\frac{2 \pi e^{2}}{q \varepsilon(\omega)}
$$

where

$$
\varepsilon(\omega)=\varepsilon_{\infty} \frac{\omega_{l}^{2}-(\omega+i \Gamma)^{2}}{\omega_{t}^{2}-(\omega+i \Gamma)^{2}}
$$


Here $\varepsilon_{\infty}$ is the lattice dielectric susceptibility for $\omega \rightarrow \infty, \omega_{l}\left(\omega_{t}\right)$ are the limiting frequencies of the longitudinal (transverse) optic phonons while $\Gamma$ is the phonon damping due to the phonon anharmonicity.

Equation (3) describes the direct interaction between two electrons. One should, however, also allow for the indirect interaction where the first electron interacts with the second one and this, in its turn, interacts with the next electron. Taking this in consideration, one should take into account the following two points. First, one can consider any electron as the next one. This will give the factor $n_{s}$. Second, the interaction we are discussing is of a resonant nature. In the 2D case the electron spectrum, unlike the 3D case, has no component of the quasimomentum along the magnetic field. As a result, the characteristic time of e-e interaction is not $m / \hbar q_{z}^{2}$ as in $3 \mathrm{D}$ case but is determined by $1 / \Gamma_{e}$ where $\Gamma_{e}$ is the electron damping. This means that the electrons will be in resonance during the time of the order of $1 / \Gamma_{e}$. As a result, we get for this interaction Eq. (2) with an extra factor [6]

$$
\frac{2 \pi e^{2}}{q \varepsilon(\omega)} \cdot \frac{n_{s}}{\hbar\left(\omega-\mathcal{N} \Omega \cos \theta+i \Gamma_{e}\right)} .
$$

The whole expression is dimensionless.

Now we should take into account that this interaction may take place $1,2,3, \ldots$ times. As a result, the full interaction is

$$
V_{\text {full }}=\frac{2 \pi e^{2}}{q \varepsilon(\omega)}\left[1-\frac{n_{s}}{\hbar\left(\omega-\mathcal{N} \Omega \cos \theta+i \Gamma_{e}\right)} \cdot \frac{2 \pi e^{2}}{q \varepsilon(\omega)}\right]^{-1} .
$$

The interaction becomes very strong provided the expression in the square brackets vanishes. In fact this condition means the existence of electron transitions between Landau levels due to the interaction with the mixed electron-phonon mode.

We will solve the equation

$$
1-\frac{n_{s}}{\hbar\left(\omega-\mathcal{N} \Omega \cos \theta+i \Gamma_{e}\right)} \cdot \frac{2 \pi e^{2}}{q \varepsilon(\omega)}=0
$$

by iterations considering the dampings as relatively small. In the lowest approximation we have two solutions we are interested in

$$
\omega=\mathcal{N} \Omega \cos \theta, \text { and } \quad \omega=\omega_{t}
$$


It means that

$$
\omega_{t}=\mathcal{N} \Omega \cos \theta
$$

This condition determines the MPR peak positions. The next, imaginary, approximation determines the width of the $\mathcal{N}$ th MPR peak

$$
\Gamma_{\mathcal{N}}=\Gamma_{e}+\frac{n_{s}}{n_{\mathrm{up}}} \Omega \cos \theta
$$

where

$$
n_{\mathrm{up}}=\frac{\varepsilon_{\infty} \hbar \Omega \cos \theta\left(\omega_{l}-\omega_{t}\right) q}{2 \pi e^{2} \Gamma}
$$

$\Gamma_{\mathcal{N}}$ should be smaller than the spacing between the Landau levels. For large concentrations $n_{s}$, in Eq. (7) the last term is predominant. This gives the condition

$$
n_{s} / n_{\text {up }} \ll 1
$$

Here we have assumed that

$$
\left(\omega_{l}-\omega_{t}\right) / \omega_{l} \ll 1
$$

and will neglect the terms proportional to this small parameter as compared to 1 . For the estimates we will take $q=q_{T} \equiv \hbar^{-1} \sqrt{2 m k_{\mathrm{B}} T}$.

When the parameter $n_{s} / n_{\text {up }}$ is of the order of 1 the MPR peaks begin to overlap and at $n_{s} / n_{\text {up }}>1$ the MPR amplitude should rapidly go down. However, if one follows the MPR maximum whereas $n_{s}$ does not depend of the magnetic field $B$ the parameter $n_{s} / n_{\text {up }}$ does not change [see Eq. (6)] and, as a result, the height of the MPR maximum remains constant. In the region $n_{s} / n_{\mathrm{up}}>1$ even small variation of $n_{s}$ due to the variation of $B$ may result in a strong variation of the height of the MPR maximum. In case the height of the MPR peak depends only of the parameter $n_{s} / n_{\mathrm{up}}$ the height would remain the same irrespective of the way we change this parameter (it can be changed either by magnetic field variation or by doping).

Let us check as to whether a relatively small concentration variation due to a small magnetic field variation 


$$
\Delta B_{\mathcal{N}}(\theta)=B_{\mathcal{N}}(0)\left(\frac{1}{\cos \theta}-1\right)
$$

is sufficient to explain the decrease of the height of the MPR maximum due to the tilting of the field $\mathbf{B}$ by the angle $\theta$. According to Ref. [4], in the relevant interval of electron concentrations under variation of $n_{s}$ by 45 per cent, i.e. from $3 \cdot 10^{11} \mathrm{~cm}^{-2}$ to $5.5 \cdot 10^{11} \mathrm{~cm}^{-2}$ the amplitude of the maximum has been decreased by a factor of 12 . This means a doubling of the amplitude due to decrease of the concentration by 2 per cent. The data given in the present paper are obtained on the samples with the carrier concentrations in this region. In Fig. 3 the amplitude of the second maximum at $170 \mathrm{~K}$ has decreased by a factor 2 at the angle $\theta_{1 / 2}=25^{\circ}$. The variation of magnetic field is $\Delta B=1.13 \mathrm{~T}$. In the perpendicular $\mathbf{B}$ the maximum is at $11.25 \mathrm{~T}$. The variation of electron concentration at this temperature is 1.5 per cent for $1 \mathrm{~T}$ (Fig. 1). Thus the increase of the concentration is 1.7 per cent at the angle of tilting $25^{\circ}$. The agreement may be considered as reasonable.

The rate of concentration variation as a function of magnetic field goes up with the temperature (see Fig. 1). If the decrease of the MPR amplitude is determined by growth of the concentration this should enhance the sharpness of the angular dependence of the MPR amplitude with the temperature. In fact this behavior has been observed in our experiment (see Figs. 3, 4, 5). Indeed, in Fig. 4 at $\mathrm{T}=190 \mathrm{~K}$ for $\mathcal{N}=2, \theta_{1 / 2}=17^{\circ}$. This corresponds to a smaller variation of the field, $\Delta B=0.6 \mathrm{~T}$. As, however, the rate of concentration variation with $B$ goes up with higher temperatures (it is of the order of three per cent per $1 \mathrm{~T}-$ see Fig. 1) one has in fact the same variation of the concentration $\Delta n_{s} / n_{s}=1.8 \%$. With our accuracy this coincides with the drop of the MPR amplitude under the variation of the carrier concentration in the perpendicular magnetic field $\mathbf{B}$ - see Ref. [4]. One has a decrease of the maximum by a factor 2 for enhancement of the concentration $n_{s}$ by 2 per cent.

We can offer the following direct experimental proof that the considered effect depends on the variation of the electron concentration $n_{s}$ in the magnetic field. In the same sample, for the same variation of the MPR amplitude it is necessary that the variation of the concen- 
tration $n_{s}$ under rotation of the sample should be the same for different values of magnetic field. In other words,

$$
\Delta n_{s}=\Delta B_{\mathcal{N}}(\theta) \frac{\partial n_{s}}{\partial B}
$$

should be $\mathcal{N}$-independent. According to Fig. 1, in the interval of the field variation $4-$ $12 \mathrm{~T}$ the concentration is with our accuracy a linear function of the field $B$. In other words, for $\mathcal{N}=2$ and $3, \Delta B_{\mathcal{N}}\left(\theta_{1 / 2}^{(\mathcal{N})}\right)$ should be $\mathcal{N}$-independent. Then, according to Eq. (9), we have for example for $\mathcal{N}=2$ and 3

$$
\cos \theta_{1 / 2}^{(3)}=\frac{B_{3}(0)}{B_{3}(0)+B_{2}(0)\left(1 / \cos \theta_{1 / 2}^{(2)}-1\right)}
$$

The sharper is the peak, the more sensitive is Eq. (10) to the variation of the angles $\theta_{1 / 2}$. As the peaks become more narrow with the temperature, we have chosen $T=230 \mathrm{~K}$. Then

for $\mathcal{N}=2$ we have $\theta_{1 / 2}=12^{\circ}$. As $B_{2}(0)=11.25 \mathrm{~T}, B_{3}(0)=7.5 \mathrm{~T}$ we get $\theta_{1 / 2}^{(3)}=15^{\circ}$ that is in a good agreement with the experimental value (see Fig. 5).

\section{CONCLUSION}

In summary, we have investigated the magnetophonon resonance in a tilted magnetic field measuring also the 2D electron concentration of the same samples. Analyzing the experimental data we have arrived at the following conclusions. The sharp angular dependence of the MPR maxima on the magnetic field is a manifestation of a very sharp concentration dependence of the MPR amplitude in the perpendicular magnetic field. The reason as to why the $2 \mathrm{D}$ concentration of the carriers can enhance is, as we understand, the following. At the tilting of the magnetic field the MPR maximum is shifted towards the strong magnetic fields \{see Eq. (11) and Fig. 6 that agrees with the data of Ref. [四] . The shift is comparatively small (of the order of $1 \mathrm{~T}$ ) and at high temperatures brings about comparatively small concentration variation (of the order of several \%). However, due to a very sharp concentration dependence of the MPR amplitude, this is sufficient for a decrease of the amplitude by several times in the relevant concentration interval. 
It would be very interesting to investigate in future the MPR in quantum wells of various compositions. It is also desirable to make a systematic investigation of the MPR in nanostructures of different forms, such as quantum wires (see, for instance Ref. [12]) as well as to take into consideration the polaron effect (cf with Ref. [13]). And of course it is important to understand the behavior of the electron concentration $n_{s}$ as a function of $T, B$ and $\theta$ in quantum wells of different compositions, doping and dimensions.

Thus the principal conclusion of the paper can be formulated as follows. The angular dependence of the MPR amplitudes as well as the decrease of the resonance widths with the temperature is a manifestation of dependence of $n_{s}$ on the total magnetic field $B$ (observed explicitly in the present paper). This statement permits to relate three seemingly different groups of experiments performed in different laboratories.

1. A sharp decrease of the MPR amplitude in perpendicular magnetic field as a function of growing $n_{s}$ with a steep angular dependence of the MPR amplitude under the tilting of the magnetic field.

2. A narrowing of the angular dependence of the MPR peaks as a function of rising temperature with the enhancement of the rate of variation of $n_{s}$ as a function of $B$.

3. The characteristic width of the MPR for different $\mathcal{N}$ with the rate of variation of $n_{s}$ as a function of $B$.

We wish to emphasize that the dependence $n_{s}(B, T)$ has not been an adjustable function. Rather it has been extracted from the Hall effect measured on the same samples.

\section{ACKNOWLEDGMENTS}

V. V. A., V. L. G., M. O. S., M. A. Sh. and M. L. Sh. are grateful to the Wihuri Foundation and to the Academy of Finland (project 79543) for a partial support of this work and to the Turku University for hospitality. V. V. A. and V. L. G. also acknowledge a 
partial support for this work by the Russian National Fund of Fundamental Research (Grant 00-15-96748). 


\section{REFERENCES}

[1] V. L. Gurevich, Yu. A. Firsov, Zh. Eksp. \& Teor. Fis. 40, 199 (1961) [Sov. Phys. — JETP 13, $137(1961)]$.

[2] Yu. A. Firsov, V. L. Gurevich, R. V. Parfeniev, I. M. Tsidil'kovskii, Magnetophonon resonance. In: Landau Level Spectroscopy, ed. G. Landwehr and E. I. Rashba, Elsevier, 1991.

[3] D. C. Tsui, Th. Englert, A. Y. Cho, and A. C. Gossard, Phys. Rev. Lett. 44, 341 (1980).

[4] R. J. Nicholas, Two-dimensional magnetophonon effect. In: Landau Level Spectroscopy, ed. G. Landwehr and E. I. Rashba, Elsevier, 1991.

[5] V. V. Afonin, V. L. Gurevich, R. Laiho, Phys. Rev. B 62, 15913 (2000).

[6] V. V. Afonin, V. L. Gurevich, R. Laiho, Phys. Rev. B 65, 115301 (2002).

[7] R. Merlin, Solid State Com. 64, 99 (1987).

[8] M. A. Brummel, D. R. Leadley, R. J. Nicholas, J. J. Harris, and C. T. Foxon, Surface Sci. 196, 451 (1988).

[9] A. A. Kiselev, E. L. Ivchenko, and U. Rässler, Phys. Rev. B 58, 16353 (1998).

[10] Mark that any magnetic field dependent corrections to 2D electron Hamiltonian enter in the combination $B \cos \theta$.

[11] V. L. Gurevich, A. I. Larkin, and Yu. A. Firsov, Fiz. Tverd. Tela (Leningrad) 4, 185 (1962) [Sov. Phys. — Solid State 4, 131 (1962)].

[12] G. Ploner, J. Smoliner, G. Strasser, M. Hauser, and E. Gornik, Phys. Rev. B 57, 3966 (1998).

[13] G.-Q. Hai and F. M. Peeters, Phys. Rev. B 60, 8984 (1999). 


\section{FIGURE CAPTIONS}

1. Variation of $2 \mathrm{D}$ electron concentration $n_{s}$ as a function of magnetic field $B$ for various temperatures.

2. Variation of 2D electron concentration $n_{s}$ as a function of temperature $T$.

3. Angular dependence of the height of MPR maximum, $n_{s}=4.0 \cdot 10^{11} \mathrm{~cm}^{-2}, \mathrm{~T}=170 \mathrm{~K}$.

4. Angular dependence of the height of MPR maximum, $n_{s}=2.2 \cdot 10^{11} \mathrm{~cm}^{-2}, \mathrm{~T}=190 \mathrm{~K}$.

5. Angular dependence of the height of MPR maximum, $n_{s}=4.0 \cdot 10^{11} \mathrm{~cm}^{-2}, \mathrm{~T}=230 \mathrm{~K}$.

6. MPR maximum position as a function of the angle of tilting. The broken line corresponds to the dependence $B_{2}(0) / \cos \theta$ for $n_{s}=4.0 \cdot 10^{11} \mathrm{~cm}^{-2}$. 


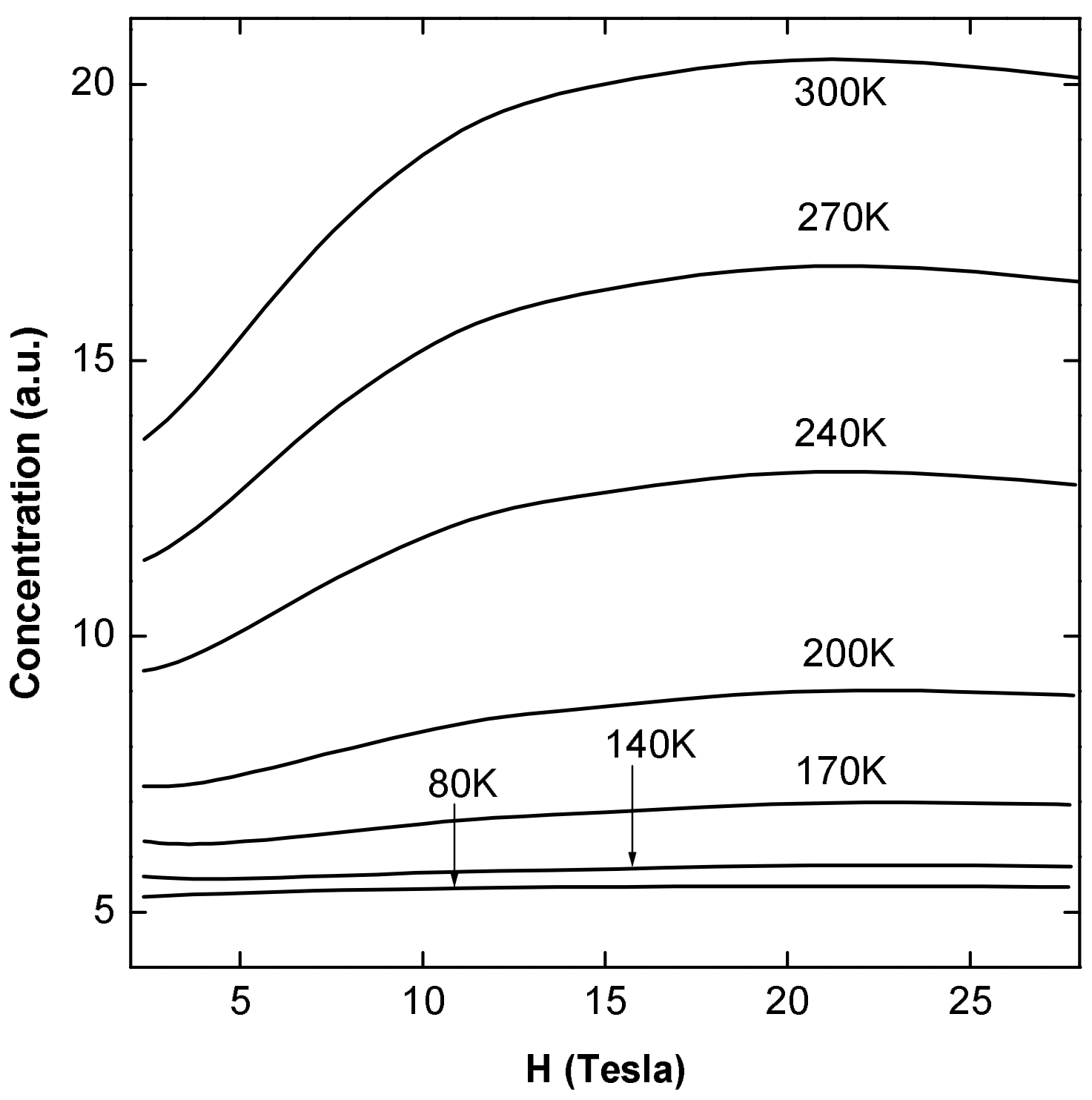




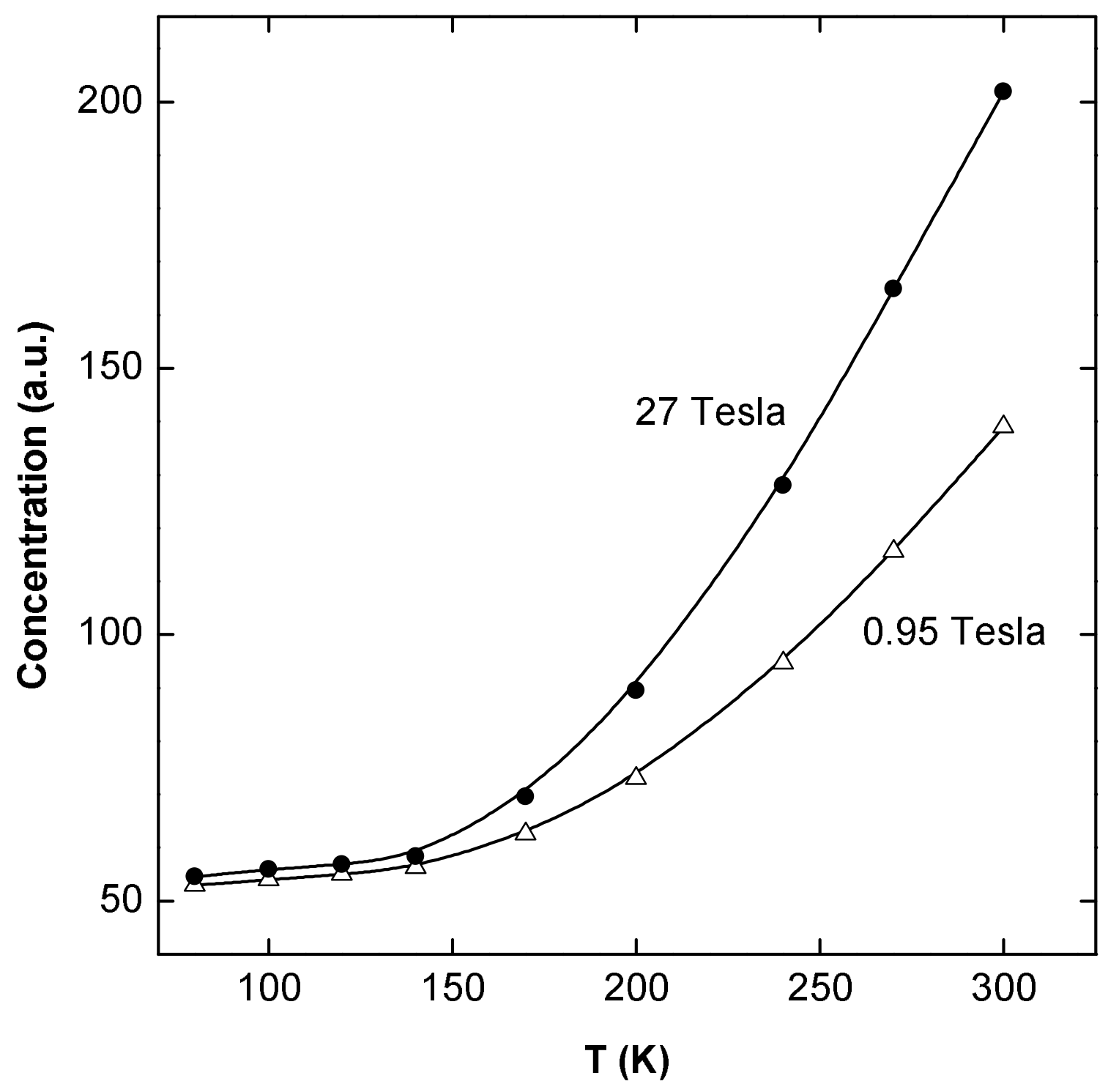




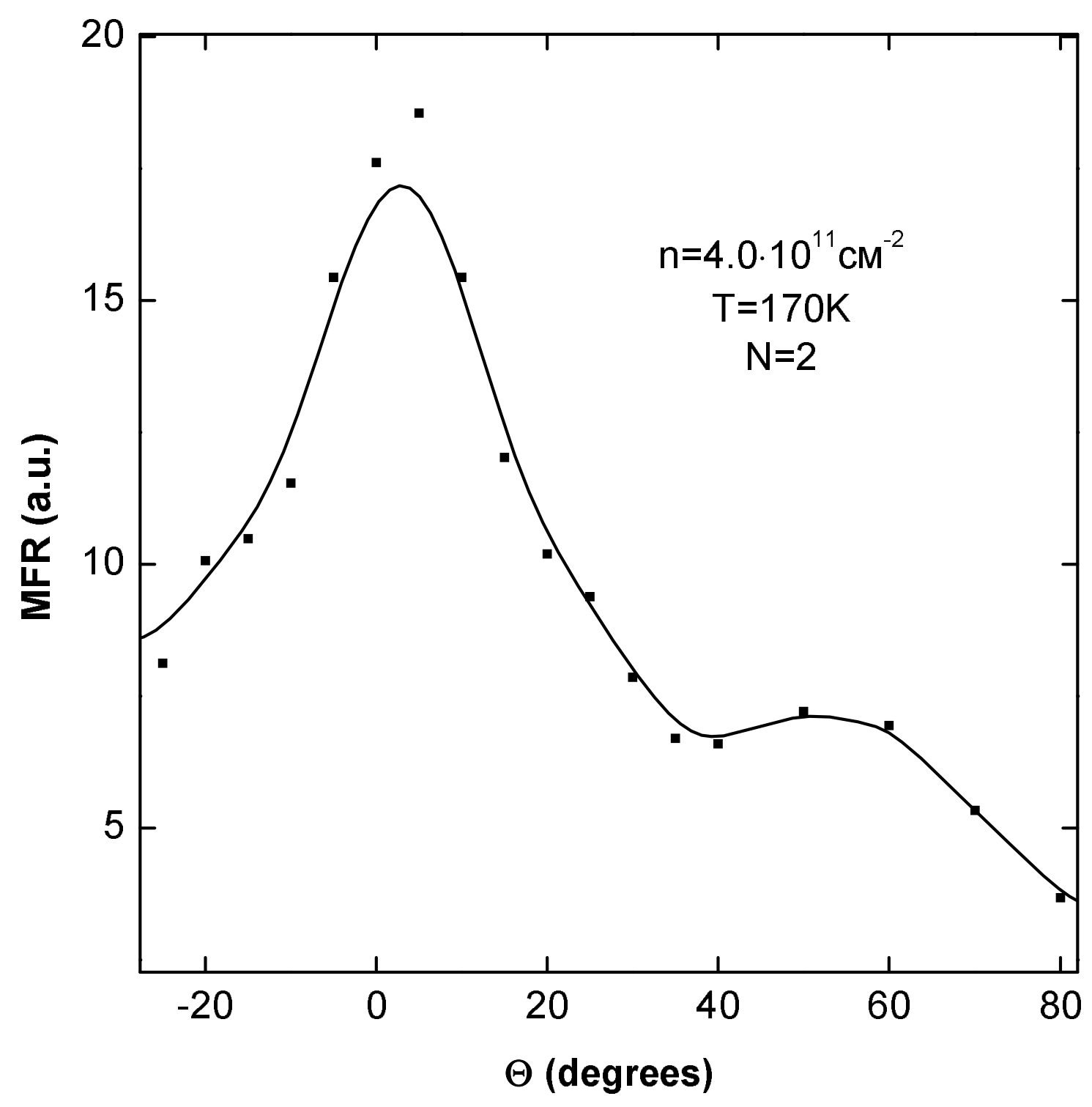




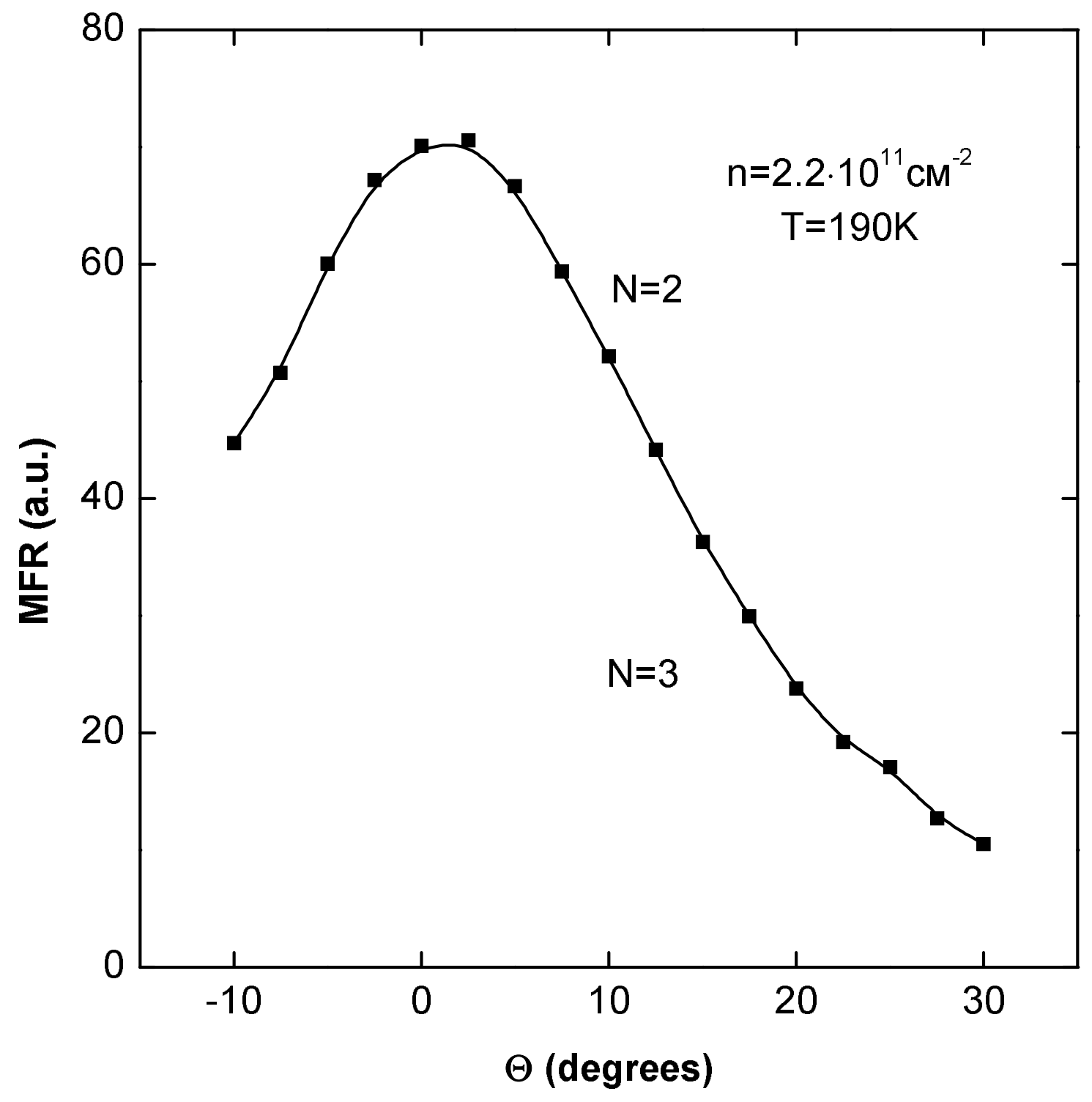




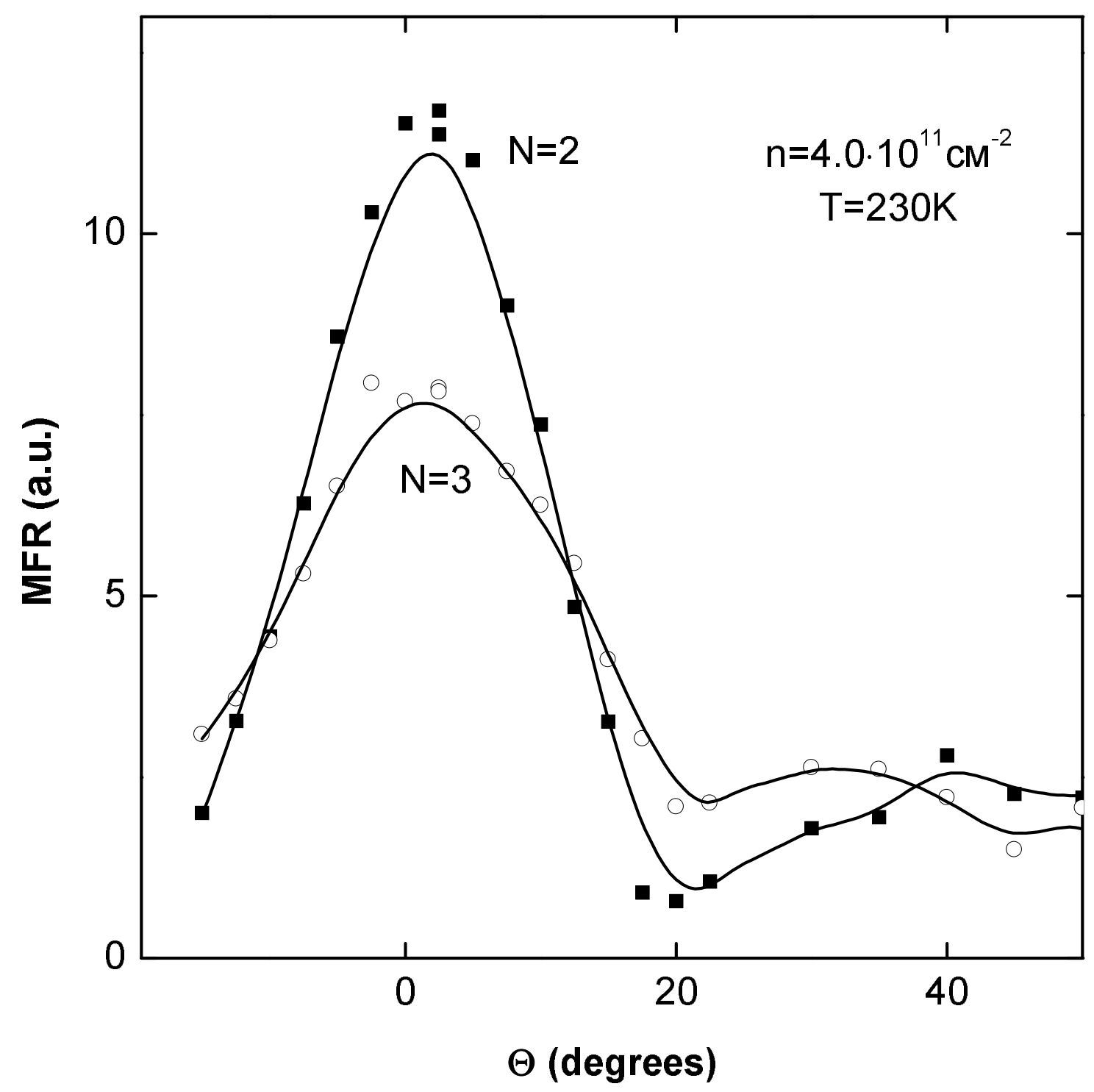




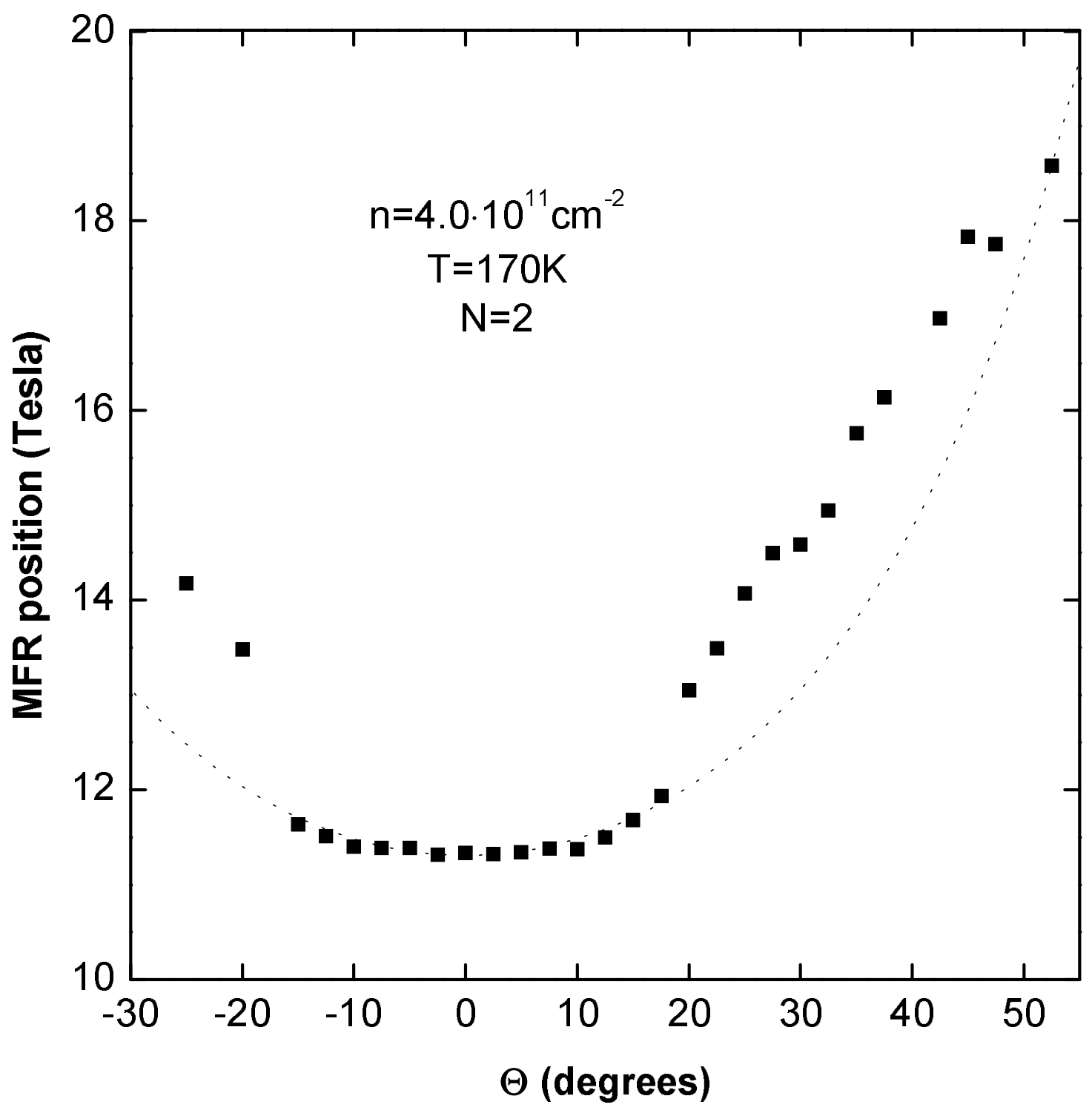

